\title{
On Applications of Matroids in Class-oriented Concept Lattices
}

\author{
Hua Mao* \\ Department of Mathematics, Hebei University, Baoding 071002, China
}

Received: 11 Jun. 2013, Revised: 7 Oct. 2013, Accepted: 8 Oct. 2013

Published online: 1 Jan. 2014

\begin{abstract}
Class-oriented concept lattices are systems of conceptual clusters, called class-oriented concepts, which are partially ordered by a subconcept-superconcept hierarchy. The hierarchical structure represents a structured information obtained automatically from the input data table. This paper presents the correspondent relations between matroids and class-oriented concept lattices. Under isomorphism, it presents necessary and sufficient conditions to discuss sub-contexts to be compatible by matroid theory. The paper also contains illustrative examples.
\end{abstract}

Keywords: matroid; class-oriented concept lattice; geometric lattice; class-oriented concept

\section{Introduction}

Formal concept analysis (FCA) is a method of exploratory data analysis that aims at the extraction of natural clusters from object-attribute data tables. It treats both the individual objects and the individual attributes as distinct entities for which there is no further information available except for the relationship saying which objects have which attributes. There are many types of binary relations between objects have been studied $([1,2])$. Equivalence relation on objects is one of them. The importance of equivalence relation on objects in some different ways has been appeared (cf.[2,3,4]). Equivalence relation can induce a partition of the universe and the clusters can construct a hierarchy order and form a lattice. One of the clusters, which is relative to class-oriented concepts, is naturally interpreted as human-perceived concepts in a traditional sense $([2,5])$.

As a branch of mathematics, matroid theory borrows extensively from the terminology of linear algebra and graph theory. It has been studied in many ways such as lattice theory and geometry approach ([6,7,8,9,10,11]). Based on its abundant theoretical contents, matroid theory has been already applied in many other fields $([6,7,11,12$, $13,14]$ ), especially, in the fields relative to FCA (cf.[15]).

This paper uses matroid theory to study on class-oriented concept lattices. Under isomorphism, we present necessary and sufficient conditions to discuss the relation between the category of simple matroids and the category of class-oriented concept lattices. Afterwards, we deal with some properties of class-oriented concept lattices for sub-contexts.

Section 2 presents preliminaries. Section 3 presents the main results and illustrative examples. Section 4 presents conclusions and an outline of future research.

\section{Preliminaries}

We assume that a data set is given in terms of a formal context (or say, a binary table) as [16]. For simplicity, in this paper, we only consider a finite set of objects and a finite set of attributes, finite matroids and finite lattices. The results may not be true for the infinite cases.

\subsection{Lattice theory}

For the looking in detail at the relations between class-oriented concept lattices and matroids, lattices especially geometric lattices will play an important role. Thus, it starts by reviewing those aspects of lattice theory. All the knowledge about lattice theory are referred to [6], [17].

\footnotetext{
*Corresponding author e-mail: yushengmao@263.net
} 
If two lattices $L_{1}$ and $L_{2}$ are isomorphic, then it will be denoted by $L_{1} \cong L_{2}$ in this paper.

Definition 2.1.1. A finite lattice $L$ is semimodular if for all $x, y \in L: x$ and $y$ cover $x \wedge y \Rightarrow x \vee y$ covers $x$ and $y$. A finite lattice is geometric if it is semimodular and every point is the join of atoms.

Lemma 2.1.1. Semimodular lattices are characterized by: (1) $L$ is semimodular if and only if it satisfies the Jordan-Dedekind chain condition and its height function $h$ satisfies for all $x, y$, (2) $h(x)+h(y) \geq h(x \wedge y)+h(x \vee y)$.

\subsection{Class-oriented formal concept lattice}

In this paper, all the knowledge about FCA come from [16]; that about class-oriented concept lattices come from $[1,5]$. In this section, we review some basic definitions of FCA and class-oriented concept lattices to be used in this paper.

Definition 2.2.1. An object-attribute data table describing which objects have which attributes can be identified with a triplet $(U, V, I)$ where $U$ is a non-empty set (of objects), $V$ is a non-empty set (of attributes), and $I \subseteq U \times V$ is an (object-attribute) relation. In $\mathrm{FCA},(U, V, \bar{I})$ is called a formal context.

Objects and attributes correspond to table rows and columns, respectively, and $(x, y) \in I$ indicates that object $x$ has attribute $y$ (table entry corresponding to row $x$ and column $y$ contains $\times$; if $(x, y) \notin I$ the table entry contains blank symbol). Based on the binary relation $I$, we can associate a set of attributes with an object. An object $x \in U$ has the set of attributes: $x I=\{y \in V \mid x I y\} \subseteq V$. Similarly, an attribute $y$ is possessed by the set of objects: $I y=\{x \in V \mid x I y\} \subseteq U$.

For each $A \subseteq U$ and $B \subseteq V$, it denotes by

$A^{*}=\{y \in V \mid$ for each $x \in A:(x, y) \in I\}$ and $B^{*}=\{x \in U \mid$ for each $y \in B:(x, y) \in I\}$.

$$
\begin{aligned}
& \text { By the above definition, it has } \\
& A^{*}=\{y \in V \mid A \subseteq I y\}=\bigcap_{x \in A} x I \quad \text { and } \\
& B^{*}=\{x \in U \mid B \subseteq x I\}=\bigcap_{y \in B} I y .
\end{aligned}
$$

Definition 2.2.2. Two objects may be viewed as being equivalent if they have the same description. An equivalence relation can be defined by for $x, x^{\prime} \in U, x \equiv_{U} x^{\prime} \Leftrightarrow x I=x^{\prime} I$.

For an object $x \in U$, the set of objects that are equivalent to $x$ is called an equivalence class of $x$ and defined by $\equiv_{U} x=\left\{x^{\prime} \in U \mid x^{\prime} \equiv_{U} x\right\}=\left\{x^{\prime} \in U \mid x \equiv_{U}\right.$ $\left.x^{\prime}\right\}=x \equiv_{U}=[x]$.

The family of all equivalence classes is commonly known as the quotient set and is denoted by $U / \equiv_{U}=\{[x] \mid x \in U\}$. It defines a partition of the universe, namely, a family of pairwise disjoint subsets whose union is the universe. A new family of subsets, denoted by $\sigma\left(U / \equiv_{U}\right)$, can be obtained from $U / \equiv_{U}$ by adding the empty set $\emptyset$ and making it closed under set union, which is a subsystem of $2^{U}$ and the basis is $U / \equiv_{U}$. The following properties hold:

(E1) $A_{1}, A_{2} \in \sigma\left(U / \equiv_{U}\right) \Rightarrow A_{1} \cap A_{2} \in \sigma\left(U / \equiv_{U}\right)$;

(E2) $A_{1}, A_{2} \in \sigma\left(U / \equiv_{U}\right) \Rightarrow A_{1} \cup A_{2} \in \sigma\left(U / \equiv_{U}\right)$.

Definition 2.2.3. A pair $(A, B), A \subseteq U, B \subseteq V$, is called a class-oriented concept if $A \in \sigma\left(U / \equiv_{U}\right)$ and $B=A^{*}$. The set of objects $A$ is called the extension of the concept $(A, B)$, and the set of attributes $B$ is called the intension.

For two class-oriented concepts $\left(A_{1}, B_{1}\right)$ and $\left(A_{2}, B_{2}\right)$, we say that

$\left(A_{1}, B_{1}\right) \leq\left(A_{2}, B_{2}\right)$ if and only if $A_{1} \subseteq A_{2} \ldots$. (F1)

The family of all class-oriented concepts forms a complete lattice called class-oriented concept lattice which is denoted by $\mathscr{B}\left(U / \equiv_{U}\right)$ in this paper. It gives a hierarchical structure of the elements in $\sigma\left(U / \equiv_{U}\right)$ and their corresponding attributes. The meet $\wedge$ and the join $\vee$ are defined by

$\left(A_{1}, B_{1}\right) \wedge\left(A_{2}, B_{2}\right)=\left(\left(A_{1} \cap A_{2}\right),\left(A_{1} \cap A_{2}\right)^{*}\right),\left(A_{1}, B_{1}\right) \vee$ $\left(A_{2}, B_{2}\right)=\left(\left(A_{1} \cup A_{2}\right),\left(B_{1} \cap B_{2}\right)\right) \ldots \ldots \ldots .(\mathrm{F} 2)$

\subsection{Matroid theory}

All the knowledge about matroid theory come from [6,7]. We only write out some of them.

Definition 2.3.1. A matroid $M$ is a finite set $S$ and a collection $\mathscr{I}$ of subsets of $S$ (called independent sets) such that (i1)-(i3) are satisfied.

(i1) $\emptyset \in \mathscr{I}$.

(i2) If $X \in \mathscr{I}$ and $Y \subseteq X$, then $Y \in \mathscr{I}$.

(i3) If $X, Y$ are members of $\mathscr{I}$ with $|X|=|Y|+1$, there exists $x \in X \backslash Y$ such that $Y \cup x \in \mathscr{I}$.

By the closure axioms (see [6], p.8, Theorem 4), a matroid $M$ is uniquely determined by the family $\mathscr{F}$ of closed sets of $M$. Thus, in this paper, we also write a matroid $(S, \mathscr{I})$ as $(S, \mathscr{F})$.

Lemma 2.3.1. Let $M=(S, \mathscr{F})$ be a matroid. Then $L(M)=$ $(\mathscr{F}, \subseteq)$ is a geometric lattice.

The correspondence between a geometric lattice $L$ and the matroid $M(L)$ on the set of atoms of $L$ is a bijection between the set of finite geometric lattices and the set of simple matroids. (The definition of $M(L)$ is referred to [6], p.52).

Based on the correspondence between $L$ and $M(L)$, for convenient, in what follows, we only use simple matroids to discuss, and besides, a simple matroid is often simply to be said a matroid. 


\section{Relations and applications}

This section will look in detail at the relationships between class-oriented concept lattices and matroids. Using these relationships, we earn that for a lattice $L$, it does not always pledge to have a formal context $\left(U_{L}, V_{L}, I_{L}\right)$ satisfying $L \cong \mathscr{B}\left(U_{L} / \equiv_{U_{L}}\right)$. In addition, under isomorphism, adopting matroid theory, we get the necessary and sufficient conditions for a sub-context to be compatible.

We begin this section to discuss the properties of classoriented concept lattices.

Lemma 3.1. The following statements about $\mathscr{B}\left(U / \equiv_{U}\right)$ are correct.

(1) $\mathscr{B}\left(U / \equiv_{U}\right)$ is a geometric lattice with $\left\{\left[x_{i}\right] \mid\left[x_{i}\right] \neq\right.$ $\left.\left[x_{j}\right],(i \neq j ; i, j=1,2, \ldots, k), \bigcup_{i=1}^{k}\left[x_{i}\right]=U\right\}$ as the family of atoms.

(2) $(A, B) \in \mathscr{B}\left(U / \equiv_{U}\right)$ if and only if $A=\bigcup_{x_{j} \in A}\left[x_{j}\right]$ and $B=\bigcap_{x_{j} \in A} x_{j} I$.

Proof. It is straightforward from (E1),(E2), (F1), (F2) and the definition of $\mathscr{B}\left(U / \equiv_{U}\right)$.

Corollary 3.1. Let $\mathscr{B}\left(U / \equiv_{U}\right)$ be given. Up to isomorphism, there is a unique matroid $M=(S, \mathscr{F})$ satisfying $(\mathscr{F}, \subseteq) \cong \mathscr{B}\left(U / \equiv_{U}\right)$. We denote this matroid as $M(\mathscr{B}(U))$.

Proof. Routine verification according to Lemma 3.1(1), the definition of $L(M)$ and the correspondence between geometric lattices and simple matroids.

By the correspondence between geometric lattices and matroids, it follows that $M(\mathscr{B}(U))$ is defined on the set of atoms of $\mathscr{B}\left(U / \equiv_{U}\right)$.

The following example shows that for a given matroid $M=(S, \mathscr{F})$, it does not pledge the existence of a formal context $(U, V, I)$ satisfying $(\mathscr{F}, \subseteq) \cong \mathscr{B}\left(U / \equiv_{U}\right)$.

Example 3.1. Let $E=\left\{x_{1}, x_{2}, x_{3}\right\}$ and $\mathscr{I}=\left\{\emptyset,\left\{x_{i}\right\},(i=1,2,3) ;\left\{x_{s}, x_{t}\right\},(s \neq t ; s, t=1,2,3)\right\}$. Then $M=(E, \mathscr{I})$ is a simple matroid with $\mathscr{I}$ as its set of independent sets. Let $\mathscr{F}$ be the family of closed sets of $M$. Then the diagram of $(\mathscr{F}, \subseteq)$ is shown in Figure 1 . Evidently, $(\mathscr{F}, \subseteq)$ is geometric.

If we suppose that there is a formal context $\left(U_{0}, V_{0}, I_{0}\right)$ satisfying $\mathscr{B}\left(U_{0} / \equiv_{U_{0}}\right) \cong(\mathscr{F}, \subseteq)$. Then it leads to the set of atoms of $\mathscr{B}\left(U_{0} / \equiv_{U_{0}}\right)$ to be $\left\{\left[x_{i}\right] \mid\left[x_{i}\right]=\left\{x_{i}\right\}, i=1,2,3\right\}$ under isomorphism. By the knowledge in Subsection 2.2, up to isomorphism, we obtain

$$
\left[x_{i}\right] \cup\left[x_{j}\right]=\left\{x_{i}, x_{j}\right\} \in \mathscr{B}\left(U_{0} / \equiv_{U_{0}}\right),(i \neq j ; i, j=1,2,3),
$$

and meanwhile,

$$
\left\{x_{1}, x_{2}, x_{3}\right\}=\left[x_{1}\right] \cup\left[x_{2}\right] \cup\left[x_{3}\right] \in \mathscr{B}\left(U_{0} / \equiv_{U_{0}}\right) .
$$

Evidently, it has $\left[x_{1}\right] \cup\left[x_{2}\right] \cup\left[x_{3}\right] \neq\left[x_{i}\right] \cup\left[x_{j}\right]$ for any $i, j \in\{1,2,3\}$. This brings the height of $\mathscr{B}\left(U_{0} / \equiv_{U_{0}}\right)$ to be 3 which is a contradiction to the construction of $(\mathscr{F}, \subseteq)$ because the height of $(\mathscr{F}, \subseteq)$ is 2 .

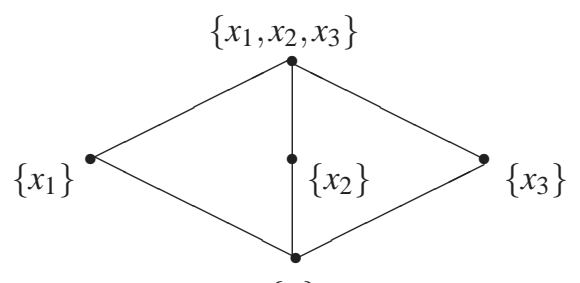

$\{\emptyset\}$

Figure 1 Diagram of $L(M)$ of the matroid $M$ from Example 3.1

Example 3.1 compels us to seek that under what conditions, a given matroid $M=(S, \mathscr{F})$ could bring about the existence of a class-oriented concept lattice $\mathscr{B}\left(U / \equiv_{U}\right)$ satisfying $\mathscr{B}\left(U / \equiv_{U}\right) \cong(\mathscr{F}, \subseteq)$.

Lemma 3.2. Let $M=(S, \mathscr{F})$ be a matroid with $\sigma_{M}$ as its closure operator and $S=\left\{x_{1}, \ldots, x_{k}\right\}$. If $M$ satisfies the following conditions,

$(M 1) \quad \sigma_{M}\left(\sigma_{M}\left(x_{i_{1}}\right) \cup \ldots \cup \sigma_{M}\left(x_{i_{t}}\right)\right)=\sigma_{M}\left(x_{i_{1}}\right) \cup \ldots \cup$ $\sigma_{M}\left(x_{i_{t}}\right)$ where $\left\{x_{i_{1}}, \ldots, x_{i_{t}}\right\} \subseteq S$;

(M2) $h\left(\sigma_{M}\left(x_{i_{1}} \cup \ldots \cup x_{i_{t}}\right)\right)=t$ for $t=1,2, \ldots, k$ where $h$ is the height function of $(\mathscr{F}, \subseteq)$,

then there exists a class-oriented concept lattice $\mathscr{B}\left(U / \equiv_{U}\right)$ satisfying $\mathscr{B}\left(U / \equiv_{U}\right) \cong(\mathscr{F}, \subseteq)$.

Proof. In light of knowledge of matroid theory, for any $x, y \in S$, it has $\sigma_{M}\left(\sigma_{M}(x) \cup \sigma_{M}(y)\right)=\sigma_{M}(x \cup y) \supseteq$ $\sigma_{M}(x) \cup \sigma_{M}(y)$. By the definition of simple matroid, for any $x \in S$, the rank of $\sigma_{M}(x)$ is 1 , that is, the set of atoms $(\mathscr{F}, \subseteq)$ is $\left\{\sigma_{M}(x) \mid x \in S\right\}$. If $\sigma_{M}(x) \neq\{x\}$ for some $x \in S$, no matter to suppose $\sigma_{M}(x)=\left\{x, y_{1}, y_{2}, \ldots, y_{n-1}\right\}$ and $n \geq 2$. It hints $\sigma_{M}(x)=\sigma_{M}\left(\sigma_{M}(x)\right)=$ $\sigma_{M}\left(\sigma_{M}\left(x \cup y_{1} \cup \ldots \cup y_{n-1}\right)\right) \subseteq S$ and $h\left(\sigma_{M}(x)\right)=1$.

But by $(\mathrm{M} 2), h\left(\sigma_{M}\left(\left\{x, y_{1}, \ldots, y_{n-1}\right\}\right)\right)=h\left(\sigma_{M}\left(x \cup y_{1} \cup\right.\right.$ $\left.\left.\ldots \cup y_{n-1}\right)\right)=n \neq 1$, a contradiction. Thus, it follows $S=\left\{\sigma_{M}(x) \mid x \in S\right\}$. Furthermore, $\sigma_{M}\left(x_{i_{1}} \cup \ldots \cup x_{i_{t}}\right)=$ $\sigma_{M}\left(\sigma_{M}\left(x_{i_{1}} \cup \ldots \cup x_{i_{t}}\right)\right)=\sigma_{M}\left(x_{i_{1}}\right) \cup \ldots \cup \sigma_{M}\left(x_{i_{t}}\right)=$ $\left\{x_{i_{1}}, \ldots, x_{i_{t}}\right\}$ holds according to the closure axioms of matroids and (M2). This implies $\sigma_{M}(X)=X \in \mathscr{F}$ for any $X \subseteq S$. Thus, $2^{S} \subseteq \mathscr{F}$ holds. However, $\mathscr{F} \subseteq 2^{S}$ is evident because the set of atoms of $\mathscr{F}$ is $S$. Therefore, $2^{S}=\mathscr{F}$ is followed.

Let $(U, V, I)$ be shown in Table 1 . From the table, we get $U=V=S=\left\{x_{1}, \ldots, x_{k}\right\}$ and

$\left[x_{i}\right]=\left\{x_{i}\right\}, x_{i} I=\left\{x_{i}\right\},(i=1, \ldots, k)$.

Hence, it is easy to prove $\mathscr{B}\left(U / \equiv_{U}\right) \cong\left(2^{U}, \subseteq\right)$.

Summing up the above, $(\mathscr{F}, \subseteq) \cong \mathscr{B}\left(U / \equiv_{U}\right)$. 
Table 1. A formal context relative to the matroid from Lemma 3.2

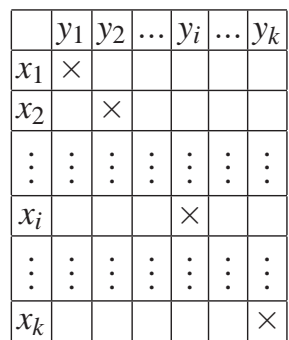

where $y_{i}=x_{i},(i=1,2, \ldots, k)$.

We easily see that a free matroid $M=(S, \mathscr{F})$, i.e. $\mathscr{I}=\mathscr{F}=2^{S}$, satisfying condition (M1) must satisfy (M2). A free matroid is trivial. Here, we pay attention to that matroids which are not free. We find out that Lemma 3.2 is satisfied by much more matroids not only free matroids.

In fact, if $M=\left(S, \mathscr{F}_{M}\right)$ is a matroid satisfying (M1) and (M2), then Lemma 3.2 hints $\left(\mathscr{F}_{M}, \subseteq\right) \cong \mathscr{B}\left(U_{M} / \equiv_{U_{M}}\right)$ for some formal context $\left(U_{M}, V_{M}, I_{M}\right)$. Corollary 3.1 points out that up to isomorphism, there exists a matroid $M\left(\mathscr{B}\left(U_{M}\right)\right)=\left(S_{U_{M}}, \mathscr{F}_{U_{M}}\right)$ satisfying $\left(\mathscr{F}_{U_{M}}, \subseteq\right) \cong \mathscr{B}\left(U_{M} / \equiv_{U_{M}}\right)$ Hence $\left(\mathscr{F}_{M}, \subseteq\right) \cong\left(\mathscr{F}_{U_{M}}, \subseteq\right)$ is right. That is to say, $M\left(\mathscr{B}\left(U_{M}\right)\right)$ is isomorphic to $M$. 3.2 .

Now we prove the truth of the converse part of Lemma

Lemma 3.3. Let $M=(S, \mathscr{F})$ be a matroid and there is a class-oriented concept lattice $\mathscr{B}\left(U / \equiv_{U}\right)$ satisfying $(\mathscr{F}, \subseteq$ )$\cong \mathscr{B}\left(U / \equiv_{U}\right)$. Then $M$ satisfies (M1) and (M2).

Proof. In view of Lemma 3.1, (E1), (E2) and (F2), it is not difficult to earn $\mathscr{B}\left(U / \equiv_{U}\right) \cong\left(2^{E}, \subseteq\right)$ where $E$ is the set of atoms in $\mathscr{B}\left(U / \equiv_{U}\right)$. Thus, this result with $\mathscr{B}\left(U / \equiv_{U}\right.$ )$\cong(\mathscr{F}, \subseteq)$ taken together follows that $M$ satisfies (M1) and (M2).

Thinking of Lemma 3.2 and Lemma 3.3 with Subsection 2.3 together, we get that up to isomorphism, every matroid which satisfies (M1) and (M2) corresponds to a unique class-oriented concept lattice, and vice versa. Thus, up to isomorphism: (1) for a matroid $M$ satisfying (M1) and (M2), we denote the correspondent class-oriented concept lattice as $\mathscr{B}(M)$; (2) for a given class-oriented concept lattice $\mathscr{B}\left(U / \equiv_{U}\right)$, we call the matroid corresponding to it as $\mathrm{CO}$-matroid and denoted it by $M(\mathscr{B}(U))$. (Actually, a CO-matroid $M(\mathscr{B}(U)$ ) is already defined in Corollary 3.1. Here is only to repeat it.)

The importance of $\mathrm{CO}$-matroids lies in the following theorem.

Theorem 3.1. The correspondence between a class-oriented concept lattice $\mathscr{B}\left(U / \equiv_{U}\right)$ and the CO-matroid $M(\mathscr{B}(U))$ on the set of atoms of $\mathscr{B}\left(U / \equiv_{U}\right)$ is a bijection between the set of class-oriented concept lattices and the set of matroids.

Subsection 2.2 shows us that each class-oriented concept lattice is a complete lattice. How about the converse part? In other words, for a complete lattice $L$, shall we find out a class-oriented concept lattice $\mathscr{B}\left(U / \equiv_{U}\right)$ such that $\mathscr{B}\left(U / \equiv_{U}\right)$ is isomorphic to $L$ ? If we want to answer this question with the definitions, it will not be easy though no answer now according to my knowledge. But if we use Theorem 3.1, we will see that the proof is quite easy. The reasons are the following:

for a complete lattice $L$, it will not pledge that it is geometric. So, owing to the corresponding between geometric lattices and matroids, it could not pledge the existence of a CO-matroid $M=(S, \mathscr{F})$ satisfying $(\mathscr{F}, \subseteq) \cong L$. Thus, it will not pledge the existence a class-oriented concept lattice $\mathscr{B}\left(U / \equiv_{U}\right)$ satisfying $\mathscr{B}\left(U / \equiv_{U}\right) \cong L$ in virtue of Theorem 3.1 and the above.

This consequence implies that there is not a "basic theorem on class-oriented concept lattices" as the one on formal concept lattices shown in [16], p.20. It also appears some difference between class-oriented concept lattices and formal concept lattices.

In the following, for $\mathrm{CO}$-matroids, we will consider its applications in the study of class-oriented concept lattices for sub-contexts. We need the following lemma.

Lemma 3.4. Let $M=(S, \mathscr{F})$ be a CO-matroid and $T \subseteq S$. Then the restriction $M \mid T$ of $M$ to $T$ is a CO-matroid.

Proof. We only need to check that $M \mid T$ satisfies (M1) and (M2) respectively according to Lemma 3.2, Theorem 3.1 and [6], p.61, Theorem 1.

Let $\sigma_{M}, \sigma_{M \mid T}$ be the closure operator of $M, M \mid T$ respectively. Let $T=\left\{x_{1}, \ldots, x_{t}\right\} \subseteq S$. Then it has $\sigma_{M \mid T}(X)=\sigma_{M}(X) \cap T$ for any $X \subseteq T$ in virtue of [6],p.61,(5).

Let $\left\{x_{i_{1}}, \ldots, x_{i_{p}}\right\} \subseteq T$. Then $\sigma_{M \mid T}\left(\sigma_{M \mid T}\left(x_{i_{1}}\right) \cup \ldots \cup\right.$ $\left.\sigma_{M \mid T}\left(x_{i_{p}}\right)\right)=\sigma_{M \mid T}\left(x_{i_{1}} \cup \ldots \ldots \cup x_{i_{p}}\right)=$ $\sigma_{M}\left(x_{i_{1}} \cup \ldots \cup x_{i_{p}}\right) \cap T=\left(\sigma_{M}\left(x_{i_{1}}\right) \cup \ldots \cup \sigma_{M}\left(x_{i_{p}}\right)\right) \cap T=$ $\left(\sigma_{M}\left(x_{i_{1}}\right) \cap T\right) \cup \ldots \cup\left(\sigma_{M}\left(x_{i_{p}}\right) \cap T\right)=$ $\sigma_{M \mid T}\left(x_{i_{1}}\right) \cup \ldots \cup \sigma_{M \mid T}\left(x_{i_{p}}\right)$. Thus, $M \mid T$ satisfies (M1).

Since $M$ is a CO-matroid, reviewing the proof of Lemma 3.2 and Lemma 3.3, we see that $A \in \mathscr{F}$ if and only if there are atoms $\left\{a_{A_{j}}: j=1,2, \ldots, n\right\}$ in $(\mathscr{F}, \subseteq)$ satisfying $A=\bigcup_{j=1}^{n} a_{A_{j}}$. (M2) hints $h_{M}(A)=n$ where $h_{M}$ is the height function of $(\mathscr{F}, \subseteq)$. In addition, the simple property of $M$ pledges $\{x\}$ to be an atom in $(\mathscr{F}, \subseteq)$ for each $x \in S$. Hence $\left\{x_{i_{j}}\right\}$ is an atom in $(\mathscr{F}, \subseteq)$ for each $x_{i_{j}} \in T,(j=1, \ldots, p)$ and $h_{M}\left(\sigma_{M}\left(x_{i_{1}} \cup \ldots \cup x_{i_{p}}\right)\right)=p$ is true. Furthermore, $\left\{x_{i_{j}}\right\}$ is an atom in $M \mid T$ also. It follows $h_{M \mid T}\left(\sigma_{M \mid T}\left(x_{i_{j}}\right)\right)=1$. We could using induction on $p$ with $\sigma_{M \mid T}\left(x_{i_{1}} \cup \ldots \cup x_{i_{t}}\right)=\sigma_{M}\left(x_{i_{1}} \cup \ldots \cup x_{i_{t}}\right) \cap T$ for $t=1, \ldots, p$, and finally, we obtain $h_{M \mid T}\left(\sigma_{M \mid T}\left(x_{i_{1}} \cup \ldots \cup x_{i_{p}}\right)\right)=p$. Thus $M \mid T$ satisfies (M2).

If one wishes to examine parts of a rather complex class-oriented concept system, it seems reasonable to exclude some objects and/or attributes from the examination. We shall describe the effects of this 
procedure on the class-oriented concept lattice. The class-oriented concept lattice of a sub-context always has an order-embedding into that of the original context. Much more information can be obtained when dealing with compatible sub-contexts, which is introduced later in this section.

If $(U, V, I)$ is a formal context and if $H \subseteq U$ and $N \subseteq V$, then $(H, N, I \cap(H \times N))$ is called a sub-context of $(U, V, I)$ (cf.[16], p.97,Definition 44).

We open the following of this section with the question of how the concept system of a sub-context is related to that of $(U, V, I)$ and how to use CO-matroids to deal with this question.

Example 3.2. Let a formal context $(U, V, I)$ be shown in Table 2 and $U=\left\{x_{1}, x_{2}, x_{3}\right\}, V=\left\{y_{1}, y_{2}, y_{3}\right\}$. Then $\left[x_{j}\right]=\left\{x_{j}\right\},(j=1,2,3)$. For a set $N=\left\{y_{1}, y_{2}\right\} \subset V$, we consider the sub-context $(U, N, I \cap(U \times N))$. In this sub-context, $\left[x_{1}\right]=\left\{x_{1}, x_{3}\right\}$ and $\left[x_{2}\right]=\left\{x_{2}\right\}$. Every extent of $(U, V, I)$ is not pledged to be an extent of $(U, N, I \cap(U \times N))$.

Table 2. Formal context from Example 3.2

\begin{tabular}{|l|l|l|l|}
\hline & $y_{1}$ & $y_{2}$ & $y_{3}$ \\
\hline$x_{1}$ & $\times$ & & $\times$ \\
\hline$x_{2}$ & & $\times$ & $\times$ \\
\hline$x_{3}$ & $\times$ & & \\
\hline
\end{tabular}

This example shows that the class-oriented concepts of a sub-context can not simply be derived from those of $(U, V, I)$ by restricting their extent and intent to a sub-context. This can be done only for compatible sub-contexts, which will be examined next.

A sub-context $(H, N, I \cap(H \times N))$ is called compatible if the pair $(A \cap H, B \cap N)$ is a class-oriented concept of the sub-context for every class-oriented concept $(A, B) \in \mathscr{B}\left(U / \equiv_{U}\right)$.

Restricting the concepts to a compatible sub-context yields a map between the class-oriented concept lattices, which necessarily has to be structure-preserving, as the following shows:

Theorem 3.2. A sub-context $(H, N, I \cap(H \times N))$ of $(U, V, I)$ is compatible if and only if $\Pi_{H, N}(A, B):=(A \cap H, B \cap N)$ for all $(A, B) \in \mathscr{B}\left(U / \equiv_{U}\right)$ defines a surjective complete homomorphism $\Pi_{H, N}: \mathscr{B}\left(U / \equiv_{U}\right) \rightarrow \mathscr{B}\left(H / \equiv_{H}\right)$.(The definition of complete lattice homorphism or complete homorphism is cf.[16], p.7,Definition 13.)

Proof. $(\Leftarrow)$ Since $\Pi_{H, N}$ is surjective, it follows $(X, Y)=$ $(A \cap H, B \cap N) \in \mathscr{B}\left(H / \equiv_{H}\right)$ for each $(A, B) \in \mathscr{B}\left(U / \equiv_{U}\right)$. Hence, $(H, N, I \cap(H \times N))$ of $(U, V, I)$ is compatible.
$(\Rightarrow)$ The first fact we need to prove is that $\Pi_{H, N}$ must necessarily be infimum-preserving and supremum -preserving for $\left(X_{j}, Y_{j}\right) \in \mathscr{B}\left(H / \equiv_{H}\right),(j=1,2)$, where $\left(X_{1}, Y_{1}\right)$ and $\left(X_{2}, Y_{2}\right)$ satisfy that it exists $\left(A_{j}, B_{j}\right) \in$ $\mathscr{B}\left(U / \equiv_{U}\right)$ satisfying $\left(X_{j}, Y_{j}\right)=\left(A_{j} \cap H, B_{j} \cap N\right)$, $(j=1,2)$.

In view of (F2), it gets:

$\left(X_{1}, Y_{1}\right) \vee\left(X_{2}, Y_{2}\right)=\left(X_{1} \cup X_{2}, Y_{1} \cap Y_{2}\right)=$ $\left(\left(A_{1} \cap H\right) \cup\left(A_{2} \cup H\right),\left(B_{1} \cap N\right) \cap\left(B_{2} \cap N\right)\right)=$ $\left(\left(A_{1} \cup A_{2}\right) \cap H,\left(B_{1} \cap B_{2}\right) \cap N\right)$, and besides, $\Pi_{H, N}\left(\left(A_{1}, B_{1}\right) \vee\left(A_{2}, B_{2}\right)\right)=\Pi_{H, N}\left(A_{1} \cup A_{2}, B_{1} \cap B_{2}\right)=$ $\left(\left(A_{1} \cup A_{2}\right) \cap H,\left(B_{1} \cap B_{2}\right) \cap N\right)$. Hence, $\Pi_{H, N}\left(\left(A_{1}, B_{1}\right) \vee\left(A_{2}, B_{2}\right)\right)=\left(X_{1}, Y_{1}\right) \vee\left(X_{2}, Y_{2}\right)=$ $\Pi_{H, N}\left(A_{1}, B_{1}\right) \vee \Pi_{H, N}\left(A_{2}, B_{2}\right)$;

$\left(X_{1}, Y_{1}\right) \wedge\left(X_{2}, Y_{2}\right)=\left(A_{1} \cap H, B_{1} \cap N\right) \wedge\left(A_{2} \cap H, B_{2} \cap\right.$ $N)=\left(A_{1} \cap A_{2} \cap H, \bigcap_{x \in A_{1} \cap A_{2} \cap H} x I \cap(H \times N)\right)$; meanwhile,

$$
\begin{gathered}
\Pi_{H, N}\left(\left(A_{1}, B_{1}\right) \wedge\left(A_{2}, B_{2}\right)\right)=\Pi_{H, N}\left(A_{1} \cap A_{2},\left(A_{1} \cap A_{2}\right)^{*}\right) \\
\quad=\Pi_{H, N}\left(A_{1} \cap A_{2}, \bigcap_{x \in A_{1} \cap A_{2}} x I\right) \\
=\left(A_{1} \cap A_{2} \cap H,\left(\bigcap_{x \in A_{1} \cap A_{2}} x I\right) \cap N\right) \in \mathscr{B}\left(H / \equiv_{H}\right) .
\end{gathered}
$$

This follows $\left(\bigcap_{x \in A_{1} \cap A_{2}} x I\right) \cap N=\left(A_{1} \cap A_{2} \cap H\right)^{*}=$ $\cap x I \cap(H \times N)$. Additionally, $x \in A_{1} \cap A_{2} \cap H$ $\Pi_{H, N}\left(A_{1}, B_{1}\right) \wedge \Pi_{H, N}\left(A_{2}, B_{2}\right)=\left(A_{1} \cap H, B_{1} \cap N\right) \wedge\left(A_{2} \cap\right.$ $\left.H, B_{2} \cap N\right)=\left(A_{1} \cap A_{2} \cap H,\left(A_{1} \cap A_{2} \cap H\right)^{*}\right) \in \mathscr{B}\left(H / \equiv_{H}\right)$ where $\left(A_{1} \cap A_{2} \cap H\right)^{*}=\bigcap_{x \in A_{1} \cap A_{2} \cap H} x I \cap(H \times N)$.

Therefore, $\Pi_{H, N}\left(\left(A_{1}, B_{1}\right) \wedge\left(A_{2}, B_{2}\right)\right)$

$$
\begin{aligned}
& =\left(A_{1} \cap A_{2} \cap H, \bigcap_{x \in A_{1} \cap A_{2} \cap H} x I \cap(H \times N)\right) \\
& =\Pi_{H, N}\left(A_{1}, B_{1}\right) \wedge \Pi_{H, N}\left(A_{2}, B_{2}\right) .
\end{aligned}
$$

The second fact we need to prove is the surjective property of $\Pi_{H, N}$.

Let $h \in H,[h]_{U}=\left\{x \in U \mid x \equiv_{U} \quad h\right\} \quad$ and $[h]_{H}=\left\{x \in H \mid x \equiv_{H} h\right\}$. Then it has

$$
\left([h]_{U}, h I\right) \in \mathscr{B}\left(U / \equiv_{U}\right)
$$

and $\left([h]_{H}, h I \cap(H \times N)\right) \in \mathscr{B}\left(H / \equiv_{H}\right)$, and further, $\Pi_{H, N}\left([h]_{U}, h I\right)=\left([h]_{U} \cap H, h I \cap N\right) \in \mathscr{B}\left(H / \equiv_{H}\right)$. Since $[h]_{U} \cap H=\{x \in H \mid h I=x I\}$

and $[h]_{H}=\{x \in H \mid x I \cap(H \times N)=h I \cap(H \times N)\}$. In addition, $\left([h]_{U} \cap H, h I \cap N\right) \in \mathscr{B}\left(H / \equiv_{H}\right)$ hints $[h]_{U} \cap H=\bigcup_{a \in[h]_{U} \cap H}[a]_{H}$ in view of Lemma 3.1. It is obviously $h \in[h]_{U} \cap H$. This leads to $[h]_{H} \subseteq[h]_{U} \cap H$. Let $a \in\left([h]_{U} \cap H\right) \backslash[h]_{H}$. Then $[a]_{H} \subseteq[h]_{U} \cap H$ is true, i.e. $a \in H$ and $a I=h I$. From $a I=h I$ and $(H, N, I \cap(H \times N))$ is a sub-context of $(U, V, I)$, we gets

$$
a I \cap(H \times N)=h I \cap(H \times N) .
$$

Furthermore, it follows $a \in[h]_{H}$, a contradiction to $a \in\left([h]_{U} \cap H\right) \backslash[h]_{H}$. This means $[h]_{U} \cap H=[h]_{H}$. In other words, in $\mathscr{B}\left(H / \equiv_{H}\right),\left([h]_{H}, h I \cap(H \times N)\right)$ could be described as $\left([h]_{U} \cap H, h I \cap N\right)=\Pi_{H, N}\left([h]_{U}, h I\right)$.

By Lemma 3.1, for each $(X, Y) \in \mathscr{B}\left(H / \equiv_{H}\right) \backslash(\emptyset, N)$, it should have $X=\bigcup_{x \in X}[x]_{H}$ and $Y=\bigcap_{x \in X} x I$. From the above of this part, it brings about $\Pi_{H, N}\left([x]_{U}, x I\right)=$ $\left([x]_{U} \cap H, x I \cap N\right)=\left([x]_{H}, x I \cap(H \times N)\right) \in \mathscr{B}\left(H / \equiv_{H}\right)$. 
Considering this result with the result in the proof of first fact above, it follows that $\Pi_{H, N}\left(\bigcup_{x \in X}[x]_{U}, \bigcap_{x \in X} x I\right)=$ $\Pi_{H, N}\left(\bigvee_{x \in X}\left([x]_{U}, x I\right)\right)=\bigvee_{x \in X}\left(\Pi_{H, N}\left([x]_{U}, x I\right)\right)=$ $\bigvee_{x \in X}\left([x]_{H}, x I \cap(H \times N)\right)=\left(\bigcup_{x \in X}[x]_{H}, \bigcap_{x \in X} x I\right)=\left(X, X^{*}\right)=$ $(X, Y)$. That is to say, each element $(X, Y) \in \mathscr{B}\left(H / \equiv_{H}\right)$ $\backslash(\emptyset, N)$ can be described as the form $\Pi_{H, N}(A, B)=(A \cap H, B \cap N)$ for $(A, B)=$ $\left(\bigcup_{x \in X}[x]_{U}, \bigcap_{x \in X} x I\right) \in \mathscr{B}\left(U / \equiv_{U}\right)$. Additionally,

$(\emptyset, U) \in \mathscr{B}\left(U / \equiv_{U}\right)$ follows $\Pi_{H, N}(\emptyset, U)=(\emptyset \cap H, U \cap N)$ $=(\emptyset, N) \in \mathscr{B}\left(H / \equiv_{H}\right)$. Therefore $\Pi_{H, N}$ is surjective.

The third fact we need to prove is that $\Pi_{H, N}$ is infimum-preserving and supremum-preserving. This fact is easily proved by Lemma 3.1 and the above two facts.

Therefore, $\Pi_{H, N}$ is a complete homomorphism.

Taking Theorem 3.1 and Theorem 3.2 together, we can describe compatible property of a sub-context using matroid theory as follows.

Theorem 3.3. A sub-context $(H, N, I \cap(H \times N))$ of $(U, V, I)$ is compatible if and only if up to isomorphism, $M(\mathscr{B}(H))=M(\mathscr{B}(U)) \mid S_{H}$ where $S_{H}$ is the set of atoms in $\mathscr{B}\left(H / \equiv_{H}\right)$.

Proof. Let $S_{U}$ be the set of atoms in $\mathscr{B}\left(U / \equiv_{U}\right)$. By Theorem 3.1, Lemma 3.2, Lemma 3.3 and Lemma 3.4, up to isomorphism, it earns $M(\mathscr{B}(U))=\left(S_{U}, \mathscr{F}_{U}\right)$ $=\left(S_{U}, 2^{S_{U}}\right)$ and $M(\mathscr{B}(H))=\left(S_{H}, \mathscr{F}_{H}\right)=\left(S_{U}, 2^{S_{H}}\right)$, where $\mathscr{F}_{U}, \mathscr{F}_{H}$ is the family of closed sets of $M(\mathscr{B}(U)), M(\mathscr{B}(H))$ respectively because $S_{U}, S_{H}$ is the set of atoms in $\left(\mathscr{F}_{U}, \subseteq\right)$ and $\left(\mathscr{F}_{H}, \subseteq\right)$ respectively up to isomorphism. By [6], p.61, it gets

$M(\mathscr{B}(U)) \mid S_{H}=\left(S_{H}, \mathscr{F}\right)$, where $\mathscr{F}=\left\{X \cap S_{H} \mid\right.$ $\left.X \in \mathscr{F}_{U}\right\}=\left\{X \cap S_{H} \mid X \in 2^{S_{U}}\right\}$.

Because $(H, N, I \cap(H \times N))$ is a sub-context of $(U, V, I)$. Thus, by Theorem 3.2, up to isomorphism, the sub-context $(H, N, I \cap(H \times N))$ of $(U, V, I)$ is compatible if and only if a class-oriented concept $(C, D) \in$ $\mathscr{B}\left(H / \equiv_{H}\right)$ satisfies that there is $(A, B) \in \mathscr{B}\left(U / \equiv_{U}\right)$ satisfying $(C, D)=(A, B) \cap S_{H}$. In other words, the sub-context $(H, N, I \cap(H \times N))$ of $(U, V, I)$ is compatible if and only if $(C, D) \in \mathscr{F}_{H} \Leftrightarrow(C, D)=(A, B) \cap S_{H}$ for some $(A, B) \in \mathscr{F}_{U}$.

Therefore, equivalently to say, the sub-context $(H, N, I \cap(H \times N))$ of $(U, V, I)$ is compatible if and only $M(\mathscr{B}(U)) \mid S_{H}=M(\mathscr{B}(H))$ holds under isomorphism.

\section{Conclusions and future research}

We present conditions for FCA for the output class-oriented concept lattices up to isomorphism. The most importance in this paper is that we provide the correspondent relations between matroids and class-oriented concept lattices. This is the foundation for the application of matroid theory to class-oriented concept lattice theory, and vice versa. Theorem 3.3. implies that matroid theory could be used to deal with sub-contexts, and further, the other properties of class-oriented concept lattices. We believe that matroid theory would be used to discuss class-oriented concept lattices in decomposition parts and factors, and so on. Because of Theorem 3.1 and the properties of sub-matroids, we also assert that many algorithms in matroid theory will be used to find out the class-oriented concepts and the class-oriented concept lattices for formal contexts. These are our future research.

\section{Acknowledgement}

The author acknowledges the financial support by NSF of China $(11101115,61202178,61073121)$, NSF of Hebei Province (A2013201119, F2012402037) and SF of Baoding (12zr035).

The author is grateful to the anonymous referee for a careful checking of the details and for helpful suggestions that improved this paper.

\section{References}

[1] Y. Yao, On generalizing rough set theory, In: Wang G. et.al. (Eds.), Proceedings of the 9th International Congerence on Rough Sets, Fuzzy Sets, Data Mining, and Granular Computing, LNAI 2639, Chongqing, Springer, (2003).

[2] Y. Chen, Y. Yao, Information Science, 178, 1-20 (2008).

[3] R. Bělohlávek, V. Sklenář, J. Zacpal, Concept lattices constrained by equivalence relations, In: V.Anásel, R.Belohlávek (Eds.), Proceedings of the CLA 2004 International Workshop on Concept Lattices and Their Applications, Ostrava, (2004).

[4] R. Bělohlávek, V. Sklenár, J. Zacpal, Concept lattices constrained by systems of partitions, In: Proc. Znalosti 2005, 4th Annual Congerence, Stara Lesna, (2005).

[5] I.Düntsch, G.Gediga, Proceedings of the 2002 IEEE International Conference on Data Mining, IEEE Computer Society, (2002).

[6] D. Welsh, Matroid Theory, Academic Press Inc., London, (1976).

[7] H. J. Lai, Matroid Theory, Higher Education Press, Beijing, (2002). (in Chinese)

[8] H. Mao, Acta Math. Sinica (Chinese series), 51, 109-114 (2008).

[9] H. Mao, Ars Comb., 81, 23-32 (2006).

[10] H. Mao, Ars Combinatroia, 90, 245-256 (2009).

[11] J. Oxley, Matroid Theory, Oxford University Press, New York, (1992).

[12] N. White, Matroid Application, Cambridge University Press, Cambridge, (1992).

[13] J. Graver, B.Servatius, H.Servatius, Combinatorial Rigidity, Amer. Math. Soc., Providence, (1993).

[14] N. Kashyap, SIAM Dis. Math., 22, 256-272 (2008). 
[15] H. Mao, Math. Comm., 14, 425-432 (2009).

[16] B.Ganter, R.Wille, Formal Concept Analysis: Mathematical Foundations, Springer-Verlag, Berlin, (1999).

[17] G. Birkhoff, Lattice Theory, third ed., American Mathematical Society, Providence, (1967).

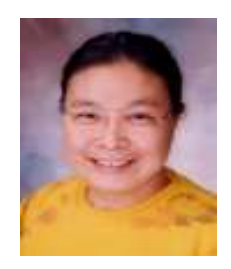

Hua Mao is a mathematics professor at Hebei University,China. She received the $\mathrm{PhD}$ degree in Applied Mathematics at Xidian University. Her research interests are in the areas of pure and applied mathematics including matroid theory, lattice theory, concept lattice theory and information science. She has published research articles in many international journals of mathematical and information sciences. 